\title{
Balance - Grundgesetz und Vollendung der Bewegung
}

Kilian Dräger

\author{
Der Mensch lebt, d. h. bewegt sich multidimensional und adaptiert sich ständig an \\ Einwirkungen unterschiedlichster Kräfte. Balance ist der Schlüssel zur Analyse und \\ Ausführung von Bewegung 1. Ordnung und höherer Ordnungen. Das Balance-Kon- \\ zept bietet erweiterte Möglichkeiten für Diagnostik (Analyse von Bewegungsmus- \\ tern, Balance-Test) und Behandlung. Dysfunktionen, d. h. Hyper- und Hypoaktivität, \\ können reguliert und auf eine individuelle Norm, die Balance, kalibriert werden.
}

\section{Prinzip der Balance}

Bei allen geglückten Bewegungen kann man das Prinzip der Balance erkennen. Geglückt sind äußere wie innere „Bewegungen“, ob sie nun bewusst oder unbewusst ablaufen, wenn sie zu Frieden und Wohlgefühl führen, was Gesundheit bedeutet. Professionelles Vorgehen verlangt die Analyse und das Verständnis dieses essenziellen Prinzips.

Das Balance-Konzept vereinfacht und erweitert zugleich das diagnostische und therapeutische Potenzial und beschreibt das Grundgesetz der Bewegung. Es vereinfacht und strukturiert die Analyse von Bewegung mit ihren grundlegenden Parametern: Kraft und Richtung (Vektor). Die Vereinfachung eröffnet ein Verständnis und Erleben der eigenen Handlungen mit der Möglichkeit einer tiefgreifenden Kalibrierung auf ein „Normal-Null“, sodass in komplexen Abläufen ungewollte Bewegungen entdeckt und beseitigt werden können.

Bewegungen werden in ihrer Aktion nach Kraft und Richtung analysiert, wobei die Art des komplexen Zusammenspiels aller Bewegungen über Funktion oder Dysfunktion entscheidet. Balance stellt dabei den wesentlichen Schlüssel zur Analyse und zur präzise ausgeführten Aktion dar. Balance ist das Grundgesetz der Bewegung. In diesem Zusammenhang sind alle funktionellen Prozesse des Menschen als Bewegung zu verstehen. Menschliche Bewegung drückt sich nicht nur als funktioneller Prozess in der Biomechanik, sondern auch in metabolisch-biochemischen Prozessen, biophysikalischen Abläufen, immunitären Vorgängen, psycho-mentalen Prozessen sowie unzähligen zellulären Dynamiken aus [1].

\section{Balance bei gesunden und krankhaften Bewegungsmustern}

Alle diese Bewegungen in den verschiedenen Dimensionen stehen in wechselseitiger Beziehung ( $\bullet \mathbf{A b b} . \mathbf{1})$ und stellen als Prozess eine multidimensionale Bewegung dar. Ist diese in balancierter Dynamik, bedeutet dies Gesundheit.

Sind die Systeme der verschiedenen Dimensionen in Dysbalance, bedeutet dies Krankheit. Doch stellt die dysfunktionale Aktivität auch einen Kompensationsversuch des wechselwirkenden komplexen Systems dar (z. B. Fieber bei bakteriellem Infekt, um die Reaktionsgeschwindigkeit des Körpers gegenüber den Bakterien zu verbessern, oder der Randzackenanbau an einem arthrotisch veränderten Gelenk, um Druck pro Fläche zu reduzieren). Die Ausbildung des Musters ist ein Versuch, die Integrität des Menschen zu erhalten ( $\boldsymbol{\sim}$ Abb. 2). Daher ist auch das dysfunktionale Muster einer Krankheit Bestandteil einer globalen Balance des Menschen - Balance ist immer Voraussetzung des Lebens.

\section{Messung der Dysbalance}

Die komplexen Wechselwirkungen erfordern eine global und lokal orientierte Analyse systemischer Ordnung, um Dysbalancen ausmachen zu können und den richtigen Ansatz für die Behandlung zu finden. Wie in allen medizinischen Fachrichtungen braucht man dazu Wissen, d.h. Kenntnis des Normalen und dessen Abweichungen. Doch reicht dies für komplexe Systeme nicht aus, da es immer Lücken in der Kenntnis menschlicher Systeme gibt. Es braucht weiterhin eine integrative Ergänzung durch emphatische Wahrnehmung des Gesamten. Eine rationale Lösung für diese besondere Aufgabe bietet das BalanceKonzept. Es bezieht das eigene wahrnehmende Erleben mit ein und nutzt die eigene Person als Messinstrument, wodurch eine außergewöhnliche Kongruenz von Logik 
und Perzeption hergestellt wird. Stete Übung ist nötig, damit der Osteopath nicht eigene Dysbalancen ( $\bullet$ Abb. 2) auf den Patienten überträgt, sondern eine balancierte Verbindung eingehen kann. Je tiefer man die eigene Balance frei herstellen kann, desto besser können auch kleinste Dysfunktionen einen in Bewegung versetzen und in der eigenen Perzeption erscheinen.

\section{Balance mehrdimensionaler Bewegungen}

Die Osteopathie ist ein wissenschaftlicher Sonderfall. Ist sie doch die einzige Wissenschaft, die ein Messinstrument einsetzen kann, das zu 100\% deckungsgleich mit dem zu untersuchenden Objekt ist. Die Osteopathie bezieht sich auf alle Ebenen im Menschen, wobei diese alle mit dem Messinstrument in Resonanz treten können, da es über genau die gleichen Ebenen verfügt wie das Gegenüber. Das erklärt auch die Notwendigkeit, dass man sich, nämlich als Messinstrument, eichen bzw. kalibrieren muss. Ohne einen verlässlichen Nullpunkt auf den verschiedenen Ebenen kann man nicht professionell tätig sein. Das braucht Zeit, weswegen die 4- bis 5-jährige Ausbildungszeit in der Osteopathie als angemessen zu sehen ist. Ähnliches vollziehen Psychotherapeuten in langjährigen Weiterbildungen im psychisch-seelischen Bereich.

Jede Bewegung ist limitiert und hat unterschiedlich geartete Grenzen, die durch anatomische, physiologische sowie auch dysfunktionale Gegebenheiten bestimmt werden.

- Abb. 3 zeigt das Schema einer eindimensionalen Bewegung 1. Ordnung.

- Erweitert man eine eindimensionale Bewegung um (eine) weitere Dimension(en), erhält man eine komplexe Bewegung, die eine Bewegung höherer Ordnung ist, weil sie mehrere Dimensionen in sich vereint, z. B. die 3 Dimensionen des Raumes mit zusätzlicher zeitlicher Dimension sowie affektive Qualitäten im psycho-mentalen Raum.

- An komplexen Bewegungsmustern sind alle menschlichen Dimensionen ( $\triangleright$ Abb. 1) beteiligt, was sie zu Bewegungen höherer Ordnung macht, bei denen es wechselseitige Bezüge gibt. Diese werden in Physiologie, Anatomie, Psychosomatik etc. wissenschaftlich dargestellt. Insbesondere hat aber jeder Mensch die Möglichkeit, diese „objektiven“ Informationen subjektiv zu erleben und intersubjektiv zu überprüfen.

Die Osteopathie hat den Anspruch, dem Patienten auf allen Dimensionen bzw. Ebenen zu begegnen und Bewegungen höherer Ordnung zu nutzen, was eine offene und freie Bewegungsmöglichkeit des Osteopathen voraussetzt. Charakteristisch für die Osteopathie ist, dass sie Bewegungen als Zusammenspiel aller Systeme ansieht und den therapeutischen Zugang zum Patienten an dessen individuelle Situation anpasst.

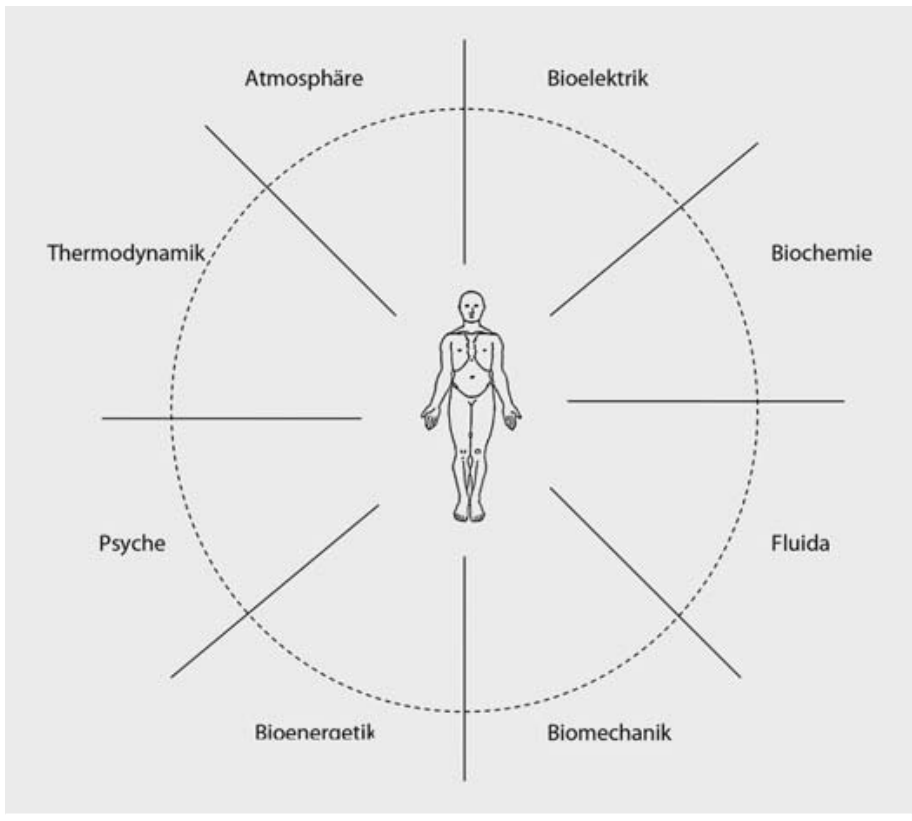

- Abb. 1 Der Mensch besteht aus verschiedenen systemischen Funktionsbereichen, deren unterschiedliche Dimensionen in Wechselwirkung stehen. Sind die Prozesse der verschiedenen Dimensionen balanciert, laufen körperlich und seelisch geistige Prozesse physiologisch ab, ist das Leben vital und gesund. (aus: [1])

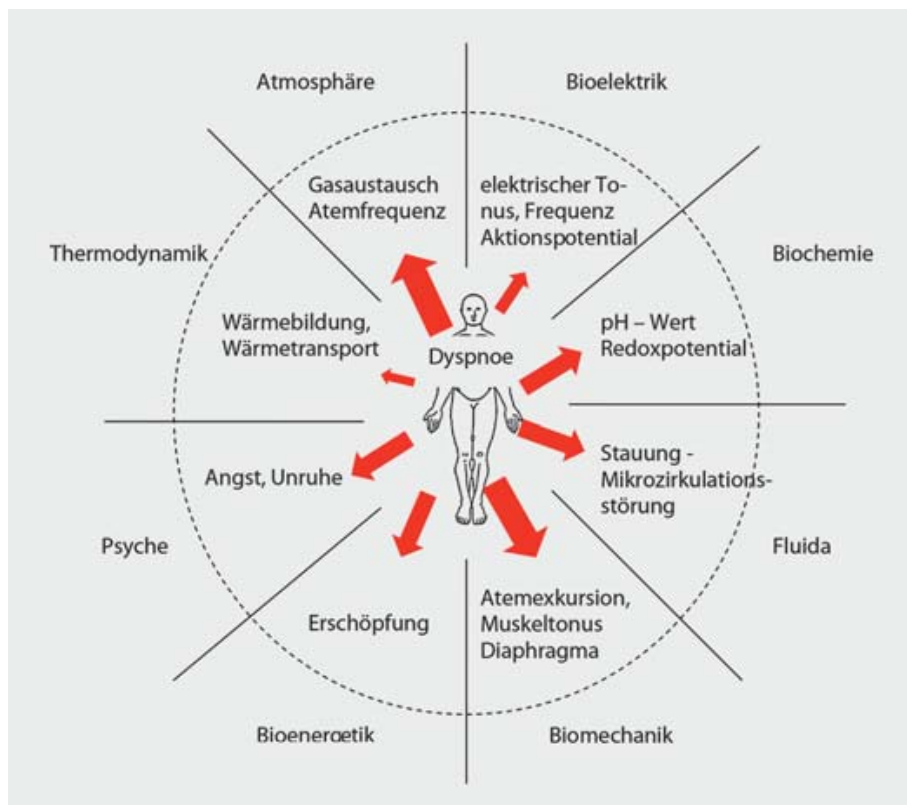

- Abb. 2 Eine Dysbalance der einzelnen Systeme bedeutet Krankheit. Das dysfunktionale Muster ist aber auch eine Kompensation zum Erhalt der Integrität und stellt damit ebenfalls eine Balance dar. (aus: [1])

Durch die eigene Freiheit kann der Osteopath im Zusammenklang mit dem Patienten eine Balance induzieren, die dem therapeutischen Prozess die Potenz verleiht. Daher ist es wichtig, für die eigene Freiheit zu sorgen, um so auf allen Ebenen Resonanz bieten zu können. Man sollte 


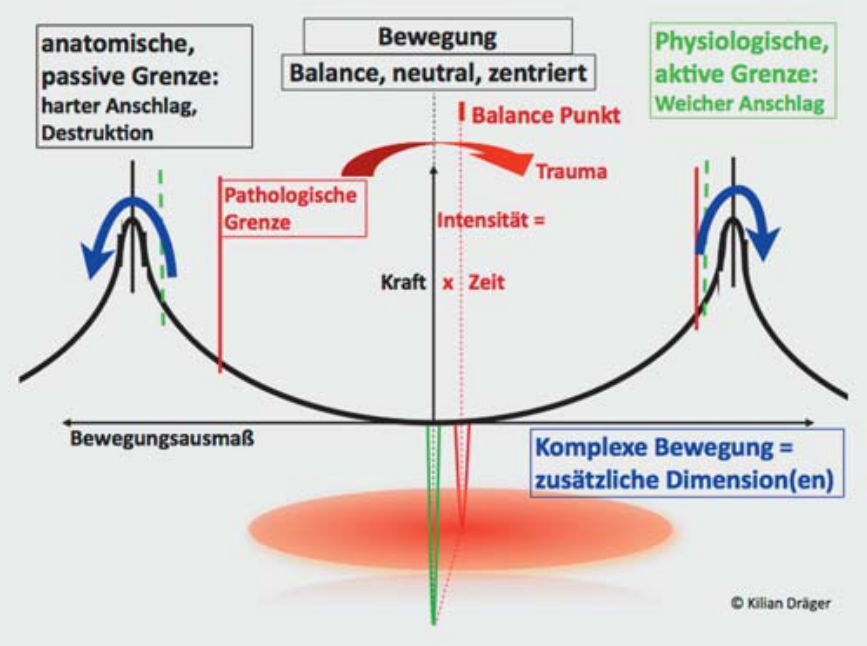

- Abb. 3 Schema einer Bewegung und ihre Grenzen mit Darstellung von Strecke/Ausmaß und Kraft. Es zeigt eindimensionale Bewegungen 1. Ordnung. Das Konzept der Balance hat den Vorteil, dass man einen Punkt der Balance zwischen 2 Bewegungspolen bestimmen kann, und daher eindeutig erkennbare Richtungen der Korrektur existieren. Erweitert man eine eindimensionale Bewegung um (eine) weitere Dimension(en), erhält man eine komplexe Bewegung (blau), die als Bewegung höherer Ordnung zu sehen ist. So werden einfache Bewegungsgrenzen überwunden, wie z. B. bei der Abduktion der Schulter, die nur durch eine arthrokinematischrotatorische Komponente endgradig möglich wird. (৫ Kilian Dräger)

einzelne Ebenen (z. B. muskuloskelettale oder biodynamische) persönlich nicht zu sehr bevorzugen, sondern den gewählten Zugang allein vom Bedarf des Patienten abhängig machen. Zu fordern ist ein den Bedingungen angepasstes Vorgehen, das man treffend als ein balanciertes Vorgehen bezeichnen kann.

\section{Das Balance-Konzept}

\section{Einfachheit und höchster Anspruch}

Das Balance-Konzept ermöglicht die Erkenntnis eines globalen dysfunktionalen Grundmusters, das auf allen Ebenen stattfindet, aber oftmals trotz großer Anstrengungen im Leben bisher nicht gelöst werden konnte. Durch das Verstehen des Grundgesetzes der Balance gibt es die Möglichkeit, die entscheidenden Änderungen mit einer Bewegung niederer Ordnung und auf einer „harmlosen“ Ebene einzuführen. Dies kann z. B. das Weglassen von Widerstand in einem Bein sein, bei dem man erlebt, dass dies der gleichgeartete Widerstand ist, den man sonst z. B. seinem Partner entgegensetzt. Wichtig sind dafür Wahrnehmungsfähigkeit und Orientierung für ökonomischen Einsatz der eigenen Bewegung in Kraft und Richtung (Vektoren). So kann man eigene Bewegungs- und Verhaltensstrategien durchschauen und überprüfen, ob man selbst tut, was man will. Dies ist eine besondere Herausforderung, da einem die eigenen Intentionen meist durch einen Gewöhnungseffekt verborgen sind. Die Paarung von Ein- fachheit der Erfahrung und höchstem Anspruch in ihrer Präzision ist typisch für das Balance-Konzept.

\section{Effektivität und Nachhaltigkeit}

Eindimensionale Behandlungen haben eine geringere Nachhaltigkeit, da die wechselseitigen Systembezüge im rigide gebliebenen Muster eher ein Rezidiv begünstigen. Eine Behandlung wird effektiv, wenn man die zugrunde liegenden automatisierten und weit vernetzten Bewegungsmuster höherer Ordnung freisetzt. Bereinigt man das tiefer verwurzelte dysfunktionale Muster, kann Heilung nachhaltiger stattfinden.

Das Verständnis der Balance kann ein Gefühl von Normalität jenseits statistischer Mittelwerte bestimmbar machen, das nach Dräger „Geschmack der Balance“ genannt wird. Dabei sind Balance, Gesundheit, „angenehm“, keine Auffälligkeiten, Physiologie oder Normalität mehr oder weniger austauschbare Begriffe. Diese einfache Balance ist nicht mit intellektueller, rationaler Direktive auszuführen. Im Gegenteil, die intellektuelle Suche ist selbst oft das Hemmnis für die Einstellung von Normalität. Viele haben (dabei) eine eingeschränkte Wahrnehmung, sodass sie bevorzugt Abweichungen und Dysfunktionen spüren, die einen dann zurecht zum Therapeuten führen. Die Erfahrung des „Geschmacks der Balance“ versetzt den Patient dagegen wieder in die Lage, (abweichende) Bewegungsrichtungen zu erkennen, eine eventuell gewünschte Korrektur vorzunehmen und den Prozess dann aktiv in einen Automatismus zu überführen und so später mühelos im Alltag zur Verfügung zu haben. Das gilt für einfache Bewegungsebenen wie für komplexe Bewegungen von Systemen gleichermaßen.

Balance ist als unbewusster, automatisierter Prozess einfach zu fühlen und auszuführen, z. B. beim Stehen auf einem Bein. Der Automatismus ist eine wesentliche Grundlage für eine nachhaltige Verbesserung, während oft die vorherige intellektuell dominierte Suche nach Lösungen ein Hindernis darstellte. Diese Veränderung gelingt mit den sonst üblichen Maßnahmen nur schwierig oder gar nicht, da die meisten Patienten ihre Übungen nicht dauerhaft als höchste Priorität ansehen. Spätestens, wenn die Beschwerden verschwunden sind, kollidiert die Aktivität der Übung mit der Aktivität des Alltags und der Übungseifer lässt in der Regel nach. Auch wenn sportliche Aktivitäten und deren Übung einen großen Nutzen bringen, so ist es trotzdem intelligenter und wirkungsvoller, sich um die Fehler im Verhalten zu kümmern und diese wegzulassen.

\section{Diagnostik mit dem Balance-Test}

Der Balance-Test (B-Test) führt eine Kraft in ein System ein, die als Adaptationsimpuls (A-Impuls) das System anregt, sich mit einem überwiegend unbewusst zur Verfügung stehenden Muster an die neue Situation anzupas- 


\section{B-Test: Strategien}

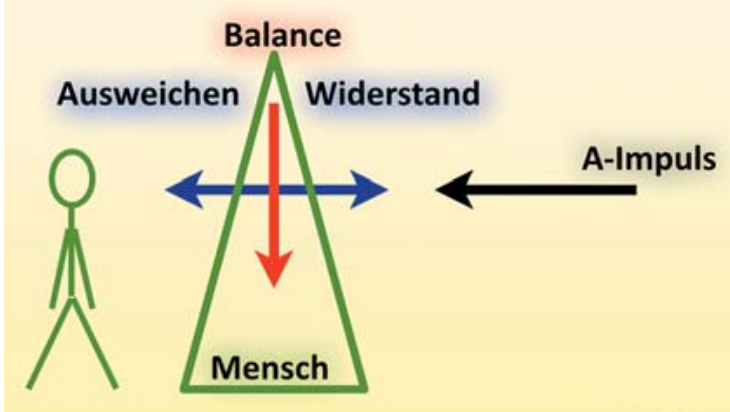

oxilun Drager

- Abb. 4 Balance-Test (B-Test): Auf einen Adaptationsimpuls (A-Impuls) können verschiedene Strategien zur Antwort genutzt werden. Aufgrund der Eindimensionalität des A-Impulses gibt es nur 3 mögliche Strategien, die hier aufgeführt sind (Ausweichen, Widerstand, Balance). Die Komplexität kommt zustande, da die verschiedenen Strategien so variantenreich kombiniert werden können, dass jeder Mensch seine individuellen Eigenheiten darin transportiert. (๔ Kilian Dräger)

sen und seine Balance aufrecht zu erhalten. Der unbewusste Vorgang nutzt automatisierte Abläufe, die in allen Bewegungsabläufen eine zentrale Rolle spielen. Diese Automatismen stellen gewohnte Bewegungsabläufe dar, die wir in unserem Leben erlernen und ursächlich am globalen Muster wie an pathophysiologischen Abläufen beteiligt sind. Der B-Test gibt Aufschluss über die Reaktion auf einen eindimensionalen Impuls und lässt gleichzeitig Rückschlüsse über Bewegungen höherer Ordnung zu.

- Der B-Test wird am stehenden Patienten mit einem dosierten eindimensionalen Schub (A-Impuls) wahlweise gegen eine Schulter oder das Becken ausgeführt.

- Wichtig ist nicht der A-Impuls, sondern die darauf folgende Antwort des Patienten. Bei der Antwort können verschiedene Dimensionen des Patienten auffällig werden, was tiefgreifende diagnostische Hinweise geben kann.
Viele Patienten fragen, was sie tun sollen, oder meinen erst, es hätte keine Alternative zu ihrer Antwort gegeben - und eben genau da liegt das Problem. Es geht um eine erweiterte Wahlmöglichkeit bei der Antwort und damit um einen freien und selbstständigen Zugriff auf die ver-

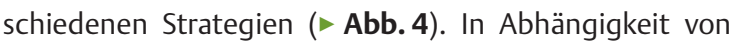
der Reaktion des Patienten kann der Osteopath dessen Stabilität und verborgene Ressourcen erkennen. Danach richten sich die folgende Behandlung und die Beratung sowie die Selbstversicherung des Patienten; so erfahren sie mehr über sich selbst und ihre Möglichkeiten.

Die meisten Patienten verstehen das Konzept leicht mittels Erklärungen auf der biomechanischen Ebene. Wichtig ist stets, dass der Patient das Verstandene in seiner Perzeption, in seiner Wahrnehmung verifizieren kann. Dies umfasst Vorgänge der körperlichen Sensorik, affektives Erleben und Kognition. Oft werden logisches Verständnis und das subjektiv Wahrgenommene nicht in Einklang gebracht. Patienten missachten die eine oder andere Seite des perzeptiven Fühlens oder logischen Denkens, aber Logik und Gefühl sollen zu einer Einheit werden. Aufgrund der Einfachheit des eindimensionalen Impulses im B-Test können die logischen Gesetzmäßigkeiten der Bewegung leicht verstanden werden und mit ihrer gleichzeitigen Perzeption verhältnismäßig leicht in Einklang kommen. Das Dilemma des Gefühls von körperlicher und geistiger Getrenntheit findet ein ersehntes und unzweifelhaftes, weil erlebtes Ende. So können Patienten Vertrauen in ihr eigenes Erleben stärken oder auch teilweise erstmals fassen. Dieses Vertrauen braucht der Patient als wesentliche Basis für die Behandlung, aber noch viel mehr für ein gutes persönliches Leben.

\section{Chancen, Angst, Mut und Vertrauen}

Das Ganze ist so bestürzend einfach und leicht verständlich einzusehen, dass es immer wieder verwundert, wieso es überhaupt Schwierigkeiten gab. Allerdings erscheint 
der Kern der Korrektur, das Weglassen der Fehler, das „Nichtstun“, als die höchste vorstellbare Herausforderung. Beim Nichtstun entsteht eher ein Gefühl der Leere und damit einer Unsicherheit. Diese wird meist mit Übersprungshandlungen ausgefüllt, mit beliebigen spontanen Aktivitäten, die zwar oft etwas Gutes intendieren, jedoch den Kern des Problems, den Fehler nicht Iösen. Die Schwierigkeit ist in den „eingefleischten“ schlechten Angewohnheiten begründet, in seltsam festen Überzeugungen und Weltbildern sowie erlebten Verletzungen. Die neue Sichtweise des Balance-Konzeptes rührt an unseren Mustern, bringt eine Eigenverantwortung als Potenzial ins Spiel und fragt nach Veränderungen, die Angst hervorrufen können. Wegen der Angst vor dem Unbekannten oder vor Schmerzen benötigt man mehr oder weniger Mut, diesem Prozess bewusst gegenüberzutreten. Aber da die Patienten das Konzept einleuchtend und notwendig sowie durch ihre Wahrnehmung bestätigt finden, können Patienten auf den zu erwartenden prozessualen Fortschritt vertrauen und so bessere Erfolge erzielen. Für das Vertrauen ist die Verbesserung der Wahrnehmung der eigenen Balance als innerer Maßstab essenziell und sollte durch kompetente Fremdwahrnehmung des Therapeuten unterstützt werden.

\section{Fallbeispiel Claudicatio spinalis}

\section{Ausgangsbefund}

Ein männlicher Patient, 60 Jahre alt, klagt über eine vor 7 Monaten neu aufgetretene Lumbalgie mit ausstrahlenden Schmerzen rechts gluteal und ins rechte Bein bis zum rechten Außenknöchel. Er muss beim Gehen pausieren, die Gehstrecke liegt unter $200 \mathrm{~m}$ und nimmt im Tagesverlauf weiter ab. Die Problematik verschlechtert sich progredient. Das MRT der LWS zeigt plurisegmentale Diskusherniationen von Th12-S1, wobei die Herniation von L3-S1 Foramenstenosen verursacht: hochgradige bei L5/ S1 rechts und L3/4 links sowie mäßiggradige bei L5/S1 links, $L 4 / 5$ beidseits und L3/4 rechts. Eine periradikuläre Therapie war nicht erfolgreich, eine operative Lyse bei L5 rechts und links wurde vom Orthopäden vorgeschlagen.

Befund: BWS und LWS in Steilstellung, LWS in Lateroflexion links und Facetten tendenziell komprimiert, M. erector spinalis beidseits lumbal hyperton, llium rechts $0,7 \mathrm{~cm}$ Tiefstand, Hüften beidseits hyperton gehalten mit Dysfunktion rechts in Außenrotation und Adduktion, bei Abduktion der Hüften Leistenschmerz beidseits, Leistenkanal rechts weich.

Der ausgeprägte Befund der LWS legt nahe, dass seit langer Zeit eine Fehlbelastung vorliegt, die unbedingt beendet werden muss, um überhaupt eine Chance auf Lösung und Besserung der Problematik zu erhalten. Es erscheint sinnlos, die Diskushernien zu beseitigen und gleichzeitig die lumbalen, notwendigerweise dysfunktionalen, Bewe- gungsmuster bestehen zu lassen. Eine Lyse bei L5 würde zwar den eingeklemmten Strukturen mehr Raum geben, jedoch bestünde die Gefahr eines Rezidivs der Bandscheibenherniation, einer schwer zu beherrschenden lumbalen Instabilität mit Einklemmungspotenzial oder einer lumbalen Osteochondrose oder Spondylarthrose als gewebedynamische Abstütz- und Absicherungsfunktion mit möglicher weiterer Spinalstenose.

\section{Diagnostik mit B-Test}

Der B-Test mit einem leichten dosierten seitlichen Schub an der Schulter wurde im Stehen durchgeführt: Der Patient beantwortet den A-Impuls zuerst mit Widerstand und bei wiederholter Ausführung mit einem kreuzenden Schritt. Der zuerst auftretende Widerstand entspricht den hohen muskulären Spannungszuständen und damit einer spontanen Haltung, die von Abwehr geprägt ist. Diese Widerstandsreaktion ist bei den starken Beschwerden verständlich, aber leider kontraproduktiv. Die Tonussteigerung bei Widerstand führt dazu, dass erheblich mehr Kräfte an dieser Stelle absorbiert und damit „verkraftet“ werden müssen. Trotzdem wird dieses Muster des Widerstands alt sein, da die Ausprägung der lumbalen Läsionen darauf hindeutet, dass diese mit Fehlbelastung über eine lange Zeit „verdient“ worden sein müssen. Zudem wurde der Automatismus durch Kontrolle des Denkens ausgebremst, eine hohe intellektuelle Reflektionsleistung bremste den Automatismus. Die Wahrnehmung selbst wurde durch Denkleistungen ersetzt, was in unserer Gesellschaft keine Seltenheit ist. Auch wenn intellektuelle Leistungen wünschenswert sind, so scheint doch der Ersatz von „Messungen“ durch das Denken auch in gewissem Widerspruch zu wissenschaftlichem Verhalten zu stehen.

Dass im Nachgang der B-Test mit einem die Mittellinie kreuzendem Schritt beantwortet wurde, ist ein prognostisch gutes Zeichen, da dies der Ausführung der BalanceStrategie entspricht. Dabei werden Kräfte weitergeleitet und durchgeleitet, ohne dass sie absorbiert werden müssen. Das ist für die Gewebebelastung die günstigste Strategie. Dass diese dem Patienten zur Verfügung steht, lässt eine freie, angepasste Strategiewahl (Balance, Widerstand, Ausweichen) erreichbarer erscheinen.

\section{Intervention: Balance-Konzept}

Es erfolgte das Vertrautmachen mit dem Balance-Konzept, mit Schulung der Wahrnehmung und Steuerung von Bewegung mit Beurteilung des notwendigen, balancierten Krafteinsatzes. Priorität hat das Weglassen divergenter Kraftrichtungen, um einen optimalen Bewegungsablauf mit minimaler Belastung zu erreichen. Diese Grundsätze wurden recht schnell verstanden und umgesetzt. Hilfreich war dafür auch der hohe Leidensdruck, der durch Verständnis des Balance-Konzeptes in eine produktive Richtung gelenkt wurde. 
Die osteopathische Behandlung erfolgte mit Schwerpunkt zunächst auf biomechanischer Ebene, um muskuloskelettale dysfunktionale und hypertone Strukturen zu befähigen, wieder in den funktionalen Ablauf integriert zu werden. Dies erforderte zum Schutz der hernierten Bandscheiben vor zu viel Kraft eine präzise Dosierung. Dabei hilft es, wenn der Patient balancierend und steuernd seinen Widerstand abbauen und sogar in die Richtung der Balance mitarbeiten kann. Zudem wurden Sutherland-Techniken als Balanced Ligamentous Tension und Balanced Fluid Tension eingesetzt.

Nach insgesamt 7 Monaten, in denen der Patient in 4 Monaten 9-mal behandelt wurde, war er bei kontinuierlich zunehmender Gehstrecke und rückläufigen Beschwerden letztlich bei unbegrenzter Gehstrecke beschwerdefrei. Nach 2 weiteren Behandlungen zur Sicherung scheint das Ergebnis stabil zu sein, zumal der Patient im Heilungsverlauf Erfahrungen mit Bewegungsstrategien sammeln und sich damit ein präventives Bewegungsverhalten aneignen konnte.

\section{Fallbeispiel Reizknie}

\section{Ausgangsbefund}

Eine 24-jährige Patientin stellte sich in meiner Praxis mit Schmerzen in den Knien (rechts mehr als links) vor, die durch Belastung wie Wandern und Treppensteigen hervorgerufen wurden. Der Nacken war verspannt mit Schmerzpunkten entlang der Wirbelsäule bis zur rechten Skapula, es bestand ein störendes Gefühl an der Skapula bei Inspiration. Weiterhin fanden sich an beiden Unterarmextensoren und insbesondere am Epicondylus radialis beidseits Schmerzen bei Belastung, z. B. beim Klavierspielen, mit einem Kontrollverlust über die Finger der linken Hand.

Befund: Hüfte rechts in Innenrotations- und Adduktionsdysfunktion, Tibia rechts in Außenrotationsdysfunktion, Hüfte links in Innenrotations- und Adduktionsdysfunktion, Genu valga, leichte tanzende Patella beidseits, leichte Schwellung der Kniegelenke, Torsionsbewegung beim Aufsetzen des Spielbeins zur Standbeinphase, Dyskoordination von Hüfte - Knie - Fuß beidseits, (Umschlagspunkt der divergenten Vektoren war jeweils die Hüfte rechts und links), L1 links in posteriorer Dysfunktion, HWS ungeordnete Mittellinie mit C3-Translation links.

\section{Diagnostik mit B-Test}

Eine Reaktion auf den A-Impuls erfolgte mit Seitschritten. Diese sind durch den Beinwechsel notwendigerweise eine Kombination aus Ausweichen und Widerstand. Ein Kreuzen ist nicht möglich und die Vektor-Ausrichtung fällt schwer. Eine muskuläre Dysbalance ist schwierig auszugleichen. Die Patientin strengt sich erfolglos an, Kontrolle über ihre Bewegungsstrategie zu erlangen. Perzep- 
tion und Fühlen, inklusive emotionaler Orientierung, sind ebenfalls schwer zugänglich. Die Bewegungen werden eher vom Ausweichen bestimmt und zu weich ausgeführt und sind von Unsicherheit geprägt. Der Eindruck des BTests wird im Behandlungsverlauf verstärkt, wobei die Patientin einen allgemein ängstlichen Eindruck macht, als ob sie sich ihrer Aufgabe nicht gewachsen fühlen würde.

\section{Intervention: Balance-Konzept}

Es erfolgten eine allgemeine Schulung im Konzept der Balance, eine zur Ausrichtung der Vektoren mit Hüfte Knie - Fuß, eine der Steuerung der Vektoren beim Hinsetzen und Aufstehen, eine von differenzierter Bewegung und Ökonomie mit Nachlassen sowie eine Orientierung durch Verständnis und Bekräftigung der eigenen Wahrnehmung. Das Nachlassen hypertoner Strukturen gibt eine Erfahrung von positiver Kontrollabgabe, die das Potenzial zu physiologischem Kontrollgewinn in sich birgt. Obwohl die ängstliche Grundhaltung weiter überwiegt, entspricht die Erfahrung nicht den Befürchtungen, sodass ein Widerspruch der Erfahrung zu dem gewohnten Gedanken- und Erwartungsmuster entsteht. Dieses wird aufgezeigt und die Patientin in ihrer Perzeption unterstützt. Ein leichter Slow-Thrust [2] für L1 war bei ängstlicher Verspannung nicht möglich. L1 und Hüfte rechts wurden mit Balanced Ligamentous Tension Technik und Counterstrain-Techniken für die Hüfte behandelt.

\section{Weitere Behandlung}

Befund 1 11\%2 Monate später: Gangbild verändert mit überwiegend achsengerechtem Krafteinsatz, Aufmerksamkeit auf Balance hilft im Alltag sowohl bei Ruhe als auch in Bewegung, keine Alltagsbeschwerden mehr, auch nicht bei Belastung. Knie beidseits reizlos, keine tanzende Patella, Ligg. collaterale und cruciata unauffällig, Hüften frei, Vektoreneinsatz sicherer und weniger ängstlich, Ellenbogen und Unterarmextensoren beschwerdefrei, Nackenschmerzen deutlich besser. Es erfolgte eine Nachjustierung der präzisen Vektorführung.

B-Test: Widerstand. Auffällig ist die Verlagerung zu besserem und gewolltem Krafteinsatz. Die Stärkung von Selbstwahrnehmung und sichererem Vektoreneinsatz hat die eigene Stabilität erhöht - biomechanisch in den Knien und gleichzeitig ist die ängstliche Grundstimmung reduziert. Dies wird von der Widerstands-Strategie unterstrichen. Trotzdem bleibt eine Lücke zur Ausübung der Balance-Strategie, die aktiv geschult wird. Der Geschmack der Balance wird mit etwas Mühe eingeführt. Allerdings erfährt die Patientin durch die Balance-Strategie in der Grenzsituation bei zunehmend starkem Druck über den einfachen Kreuzschritt einen starken Komfortgewinn. Sie erlebt einen Kontrollgewinn durch Strategiewechsel, wie sie hier z. B. mit einem einfachen Kreuzschritt das drohende Umfallen umgehen kann. Diese Erfahrung von Selbstwirksamkeit wirkt potenziell in das Unbewusste hinein. Statt Angst ist nur noch eine gewisse (physiologische) Zurückhaltung spürbar. Die Änderung in diesem Bereich wird noch etwas Entwicklungszeit brauchen, die Patientin kann aber das Ziel nach eigenen Vorstellungen für sich alleine verfolgen.

\section{Fazit}

Das Balance-Konzept erweitert die diagnostischen und therapeutischen Möglichkeiten in der (osteopathischen) Behandlung. Der B-Test ermöglicht einen tiefgreifenden Einblick in die Automatismen und hintergründigen Muster des Patienten. Durch das Verständnis dieser Muster erlangt der Patient Einflussmöglichkeiten für seine Gesundung und der Osteopath erweiterte Therapieoptionen. Das kann mit dem „Geschmack der Balance“ als neuem und natürlichem Automatismus in den Alltag eingebaut werden, sodass der Therapieerfolg nachhaltiger wird. Gleichzeitig stellt die Praxis des Balance-Konzeptes ein Mittel der Prävention dar. Dabei wird der Patient ermächtigt, besser auf sich selbst zu achten und seiner Wahrnehmung zu vertrauen. Die Erfahrung von Selbstwirksamkeit und Vertrauensgewinn in die eigenen Erfahrungen und Handlungen hat ein anderer Patient wunderbar ausgedrückt: „Ich stehe ganz anders. Ich bin ganz beeindruckt von mir selbst."

Über die Autoren

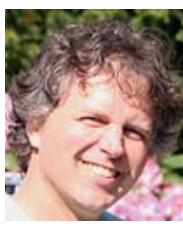

\section{Kilian Dräger}

ist Arzt, Osteopath, Physio- und Shiatsu-Therapeut. Er hat eine Dozententätigkeit für Osteopathie seit 1995, ist Fakultätsmitglied des SCCO und Lehrbeauftragter der medizinischen Fakultät der Uni Hamburg. Er setzt sich für die Osteopathie als Vorstand der osteopathischen Kindersprechstunde, der DÄGO und des BDOÄ ein. Er hat mehrere osteopathische Fachartikel und ein Buch veröffentlicht.

\section{Korrespondenzadresse}

Dr. med. Kilian Dräger D.O.

Beim Andreasbrunnen 7

20249 Hamburg

draeger@daego.de

\section{Literatur}

[1] Dräger K, van den Heede P, Klessen H. Osteopathie - Architektur der Balance. München: Elsevier/Urban \& Fischer; 2011

[2] Dräger K. Slow-Thrust-Technik. OM 2008; 9: 21-23

\section{Bibliografie}

DOI https://doi.org/10.1055/s-0043-106063

DO - Deutsche Zeitschrift für Osteopathie 2017; 15: 24-30 (c) Georg Thieme Verlag KG Stuttgart · New York ISSN 1610-5044 